1. die Abklärung, daß tatsächlich ein erhebliches Bedürfnis nach dem Reußtal als Erholungsgebiet besteht,

2. daß dieses Bedürfnis sehr vielfältig ist,

3. daß von den Besuchern der Wunsch nach verschiedenen Erholungseinrichtungen geäußert wurde.

Daraus ergibt sich die Notwendigkeit, die Kapazität bzw. die Belastbarkeit zu bestimmen, um Konsequenzen für die Erholungsplanung zu ziehen, d.h. entsprechend dimensionierte Erholungsgebiete auszuscheiden, zweckmäßige Erholungseinrichtungen bereitzustellen und Maßnahmen zu ergreifen, die eine eventuelle Überbelastung verhindern können.

\section{Literatur}

(1) Disteli M., 1969: Die Melioration der aargauischen Reußebene in ganzheitlicher Sicht, in Geographica Helvetica, Nr. 3, 1969 (S. 117).

(2) Jacsman J., 1969: Erholung am Türlersee, Arbeitsbereiche zur Orts-, Regional- und Landesplanung 1969, Nr. 8.

(3) Stauffer H. U., 1960: Was soll mit der aargauischen Reußebene geschehen? Aargauer Tagblatt v. 23.6. 60, in Bedrohte Vielfalt (S. 34).

(4) Wullschleger R., 1971: Grundlagen für eine Landschaftsplanung des aargauischen Reußtales (Diplomarbeit 1971, nicht veröffentlicht).

Adresse der Verfasserin:

Ruth Wullschleger, dipl. Natw. ETH

Kïngenmatt 48, 8055 Zürich

\title{
Das Nachdiplomstudium in Raumplanung aus der Sicht des Geographen
}

Jürg Karlen und Ulrich Roth

Seit einigen Jahren können an Schweizerischen Hochschulen Nachdiplomstudien absolviert werden, die auf einem abgeschlossenen Hochschulstudium basieren und entweder der Spezialisierung oder der Verbreiterung des Fachwissens dienen. Zur zweiten Art gehören die seit 1967 am Institut für Orts-, Regional- und Landesplanung der ETHZ durchgeführten Nachdiplomstudien in Raumpla- nung. Die Lehrziele für den laufenden Kurs sind laut Rahmenstudienprogramm:

1. Gründliche Kenntnisse der Theorien, Methoden und Verfahren der Raumplanung

2. Kenntnis der Grundzüge der für die Raumplanung bedeutungsvollen Fachgebiete im Hinblick auf die interdisziplinäre Zusammenarbeit, die 
celbständige Bearbeitung angemessener Aufgaben außerhalb des Fachgebietes des Grundstudiums

3. Fähigkeit zur Anwendung des Fachgebietes des Grundstudiums für die Raumplanung

4. Kenninis der Prinzipien interdisziplinärer $\mathrm{Zu}$ sammenarbeit und deren Anwendung im Hinblick auf die Tätigkeit als Mitarbeiter oder Leiter

\section{Kenntnis der Grundzüge der Praxis}

Die zur Verfügung stehende Zeit reicht allerdings nicht aus, um alle diese Forderungen realisieren zu können. Daher wäre diese Zielstruktur neu zu gliedern und carin Prioritäten zu setzen.

Die Erfüllung der genannten Ziele versucht man durch den folgenden Aufbau des zweijährigen Studiums anzustreben: Die ersten beiden Semester sollen dazu dienen, Kenntnisse in den Fachgebieten außerhalb des Grundstudiums mittels Vorlesungen, Seminarien und Übungen zu vermitteln. Das Unterrichtsprogramm beinhaltet die nachstehend aufgeführten Fächer:

Block Methodik: Methodik der Raumplanung Arbeits- und Führungstechnik

Block Landschaft: Landschaftsplanung und -pflege Landwirtschaft u. Kulturtechnik Forstwirtschaft

Block Siedlung: Städtebau

Architektur

Umwelthygiene

Block Mathematische Modelle und

Exakie Methoden: Systemtechnik

Statistik

Elektronische Datenverarbeitung

Block Verkehr Verkehrsplanung

und Versorgung: Verkehrstechnik

Versorgung u. Kommunikation

Block

Soziologie

Sozioökonomie: Nationalökonomie

Block Recht:

Bau- und Planungsrecht

Allgemeine Fragen des Staatsund Verwaltungsrechts

Politologie

In den zwei letzten Semestern und in den Semesterferien stehen die Gruppen- und Einzelarbeiten der Studenten im Vordergrund, wobei vor allem das interdisziplinäre Arbeiten sowie die Fähigkeit zur Anwendung des Fachgebietes des Grundstudiums für die Raumplanung geschult werden soll. Ein großes Problem beim interdisziplinären Arbeiten besteht darin, daß die Vertreter der verschiedenen
Fachrichtungen auch über verschiedene Sprachen und Methoden verfügen. Allerdings zeigt es sich, daß persönliche Präferenzen bisweilen stärker sein können als fachliche Verständigungsschwierigkeiten. Damit innerhalb der Studenten eine Interdisziplinarität erreicht werden kann, werden Absolventen folgender Fachrichtungen aufgenommen:

Architektur, Bauingenieurwesen, Forstwirtschaft, Landwirtschaft, Kulturingenieurwesen, Landschaftspflege, Geographie, Rechtswissenschaft, Soziologie, Volkswirtschaft.

In allen bisherigen Kursen war die Geographie durch 2-4 Teilnehmer vertreten.

Welche Anforderungen stellt nun der Geograph an dieses Nachdiplomstudium? Um diese Frage beantworten zu können, müssen wir zunächst die späteren Berufstätigkeiten des Geographen in der Raumplanung betrachten. Einerseits bestehen Arbeitsmöglichkeiten in der Praxis - sei es in der Privatwirtschaft oder in der öffentlichen Verwaltung -, wobei die Koordination mit anderen Fachbereichen sowie eine gute Arbeits- und Führungstechnik eine große Rolle spielen. Ein anderes Tätigkeitsfeld bietet die Forschung, in der Problemkreise wie Untersuchungen über Ökologie im weitesten Sinne als Grundlagen der Raumplanung und ihre Anwenciung, zum Beispiel bei Landschaftsplanung, -schutz und -pflege, oder die Erarbeitung von Methoden zur Lösung komplexer raumplanerischer Probleme zu behandeln sind.

Aus den vorangegangenen Ausführungen ergeben sich folgende Forderungen an das Nachdiplomstudium in Raumplanung:

1. Umfassende und ausführliche Darstellung der Zusammenhänge zwischen den Umweltwissenschaften und den Elementen der Raumplanung

2. Vorstellung und Erklärung von Methoden und ihrer Anwendung zur Untersuchung der Zusammenhänge zwischen Ökologie und Raumplanung sowie der Elemente innerhalb derselben

Diese spezifisch geographischen Forderungen beruhen auf unserer Auffassung, daß der in der Raumplanung tätige Geograph prädestiniert sein sollte, naturwissenschaftliche Probleme dieser Art zu bearbeiten und Lösungen vorzuschlagen. Darunter fallen in erster Linie Fragen der in letzter Zeit immer aktueller gewordenen Ökologie. Tschumi (1) definiert Ökologie als «die Wissenschaft von den Beziehungen zwischen den Lebewesen unter sich sowie zwischen diesen und ihrer Umwelt. Man kann sie auch als Lehre von der Ökonomie der Natur bezeichnen. Sie ist ursprünglich aus der Biologie hervorgegangen. In der heutigen, auch auf den Men- 
schen bezogenen Form ist sie aber ausgesprochen interdisziplinär und versucht, die allzulange verkannten Wechselwirkungen innerhalb der Natur sowie zwischen dieser und unserer Zivilisation zu erforschen und aufzuzeigen.»

Als weitere, allgemeine Ansprüche an das Nachdiplomstudium müssen hier erwähnt werden:

3. Der zukünftige Raumplaner ist im Hinblick auf seine spätere Tätigkeit in Arbeits- und Führungstechnik gründlich zu schulen

4. In der langfristigen Entwicklung der Gesellschaft übt der Planer eine wichtige Funktion aus. Dieser Rolle ist besondere Aufmerksamkeit zu schenken, das heißt das Bewußtsein der Verantwortung des Raumplaners gegenüber der Öffentlichkeit in seinem Handeln ist zu fördern

Aus einem Vergleich der heutigen Situation des Nachdiplomstudiums mit dem wünschbaren $\mathrm{Zu}-$ stand ergibt sich für uns unter anderem nachfolgende Kritik an der Form des Kurses:

1. Während des ersten Jahres des Nachdiplomstudiums sind die Möglichkeiten zur Weiterbildung im eigenen Fachbereich aus zeitlichen Gründen sehr beschränkt. Das wirkt sich vor allem deshalb negativ aus, weil gewisse Grundlagen, wie z. B. aus der Ökologie, von unserem Grundstudium her heute noch fehlen

2. Der Bezug zur Praxis ist zuwenig ausgeprägt. Allerdings gilt es zu bedenken, daß er in der heutigen Form des Kurses leider wohl kaum besser hergestellt werden kann

3. Die Aufgaben, die der Geograph später zu lösen hat, werden in erster Linie innerhalb der Regional- und Kantonalplanung liegen. In der Ausbildung des ersten Jahres werden aber praktisch keine Übungen und Analysen auf den Stufen Region und Kanton durchgeführt. Inwieweit die Vertiefung auf diesen Ebenen im Verlaufe der Gruppen- und Einzelarbeit erfolgen wird, kann von uns im jetzigen Zeitpunkt noch nicht beurteilt werden.

Zum Schluß unseres Vortrages wollen wir die im bisherigen Verlauf des Nachdiplomstudiums aufgetauchten hauptsächlichsten Mängel unseres Geographiestudiums an der ETHZ anführen:

Der zukünftige Geograph beschäftgt sich zu Beginn seines Studiums mit Fachgebieten wie Chemie, Zoologie, Botanik, Physik und Mathematik, die er mangels Kenntnissen seines eigenen Fachgebietes gar nicht in dieses einordnen kann. Während mehr als der Hälfte seines Studiums befaßt sich der Student mit - zum Teil für das Verständnis der Landschaftsstruktur sicher notwendigen - Nachbarwis- senschaften, um sich dann, je nach Wahlfächern, seine eigene "geographische Synthese» zusammenzubauen.

Nach Winkler (2) bedeutet «der Zweck, das letzte Ziel und der eigentliche Sinn der Geographie: Landschaftsplanung, Landschaftsgestaltung und -pflege und Landschaftsnutzung (oder auch Landes-, beziehungsweise Gebiets- und Raumplanung, -gestaltung und -nutzung), und zwar, da offenbar ein Ordnungszusammenhang zwischen den Elementen der Landschaft besteht, nicht irgendwelche, sondern bestmögliche (optimale) Landschaftsbeeinflussung».

Gerade dieser Ordnungszusammenhang zwischen den Landschaftselementen, der das Wesen der Geographie ausmacht, findet aber in der Ausbildung seinen Niederschlag nicht.

Wir möchten daher aus unserer Sicht zwei Forderungen an das Grundstudium richten:

1. Vom Beginn des Geographiestudiums an muß die Möglichkeit zum Besuche zielgerechter geographischer Spezialvorlesungen als Grundlage der Ökologie bestehen

2. Der Zusammenhang zwischen den einzelnen Landschaftselementen muß klar dargestellt werden, damit dieselben zu einer anwendbaren Umweltwissenschaft zusammengebaut werden können

Es ist uns bewußt, daß es im Rahmen dieses Vortrages nicht möglich war, ausführlich auf den gesamten Problemkomplex einzugehen. Wir bemühten uns aber, Ihnen einen Überblick über die heutige Situation des Nachdiplomstudiums in Raumplanung aus der Sicht zweier Geographen zu geben sowie Verbesserungsvorschläge zur Erhöhung der Effizienz des Studiums anzudeuten.

\section{Literatur}

(1) Tschumi P. A. (1972): Umwelt als beschränkender Faktor für Bevölkerung und Wirtschaft in: Umweltschutz und Wirtschaftswachstum. Frauenfeld 1972. S. 19.

(2) Winkler E. (1968): Von der Wissenschaftlichkeit der Geographie in: Regio Basiliensis 1968. S. 329.

Adresse der Verfasser:

Jürg Karlen, dipl. Natw. ETH

Neumattstraße 46, 5033 Buchs

Ulrich Roth, dipl. Natw. ETH

Risiweg, 5737 Menziken 